\title{
“Miracle Molecules" AI Computer-Designed, Quantum Thinking 3D Small Molecules for Brain Cancer
}

\section{John-Ioannis Grigoriadis*}

Biogenea Pharmaceuticals Ltd, Greece

*Corresponding Author: John-Ioannis Grigoriadis, Biogenea Pharmaceuticals Ltd, Greece.

Received: July 08, 2019; Published: July 10, 2019

DOI: $10.31080 /$ ASPS.2019.03.0336

\section{Background}

There are currently eleven FDA-approved small molecules to treat brain cancer, specifically Glioma and Glioblastoma, none of which have proven to be significantly effective - and in many cases minimally ineffective. The first breakthrough discovery of Galaxy AI Innovations' client, Company A, is GLYBATOMAQ, an in-silico (designed by means of computer modeling or simulation) small molecule to treat Glioma and Glioblastoma. GLYBATOMAQ far surpasses the binding properties, (the ability to attach to proteins to attack cancer cells), of all these small molecule designed to date.

To determine the number of binding sites, indicative of its ability to kill, or make healthy, more cancer cells, a proof of concept docking study employing a widely-accepted validation tool, GEMDOCK (Generic Evolutionary Method for molecular docking), a comparing GLYBATOMAQ with several of the other FDA drugs approved for use against Glioblastoma.

The results are impressive. Comparison against one particular drug, Depatuxizumab, for example showed a Docking fitness of only 50.3895. GLYBATOMAQ scored 2.54e+011 Docking fitness 594 million times higher binding affinities.

Galaxy AI Innovations has entered an exclusive agreement with Company A to sell its "Miracle Molecules", which address a range of potential indications. At this juncture, we are seeking to first sell two in-silico designed small molecules, GLYBATOMAQ and QuantumMM Microcrylaq (for treatment of non-small cell lung tumor). We are also selling.

Biogenetoligandorol, the proprietary drug design process responsible for the formulation of an additional 180 in-silico small molecules in the development pipeline.

\section{Groundbreaking innovations}

Miracle Molecules' creator, and founder of Company A is a pharmacist/inventor with unsurpassed expertise in computer-aided drug designing. His breakthrough discoveries have garnered over 15 "Best Poster Awards" around the globe.
Biogenetoligandorol, a proprietary process using artificial intelligence and related algorithms to vastly accelerate drug development. Our first product offering, GLYBATOMAQ, is a patented drug named by IUPAC. It is an in-silico hyper-molecule with anti-cancer properties aggressive against brain cancers (Glioma and Glioblastoma). In addition, Company A holds a patent for the Quantum Chemistry Methods and Software Techniques responsible for GLYBATOMAQ. The final design of such effective small molecules is a proprietary breakthrough discovery using state-of-the-art artificial intelligence and quantum computing validated using GEMDOCK as the end standard.

\section{Biogenetoligandorol features and benefits}

There is a clear opportunity for quantum computing to outpace the existing computing method and improve results; to take a hybrid approach and weight different molecule variables for comparison, a clear advantage over the traditional "black-box" comparison model. Miracle Molecules are designed using a proprietary process that abbreviates the development by which drugs are currently produced; and,

- Uses newly-available quantum hardware platforms and software application programming interfaces (APIs) using deep learning to predict how molecules will behave and how likely they are to bind together and how strongly

- Employs proprietary software that teaches itself about molecular interactions by identifying patterns and learning to recognize images, a new, advanced technology that makes discovering new drugs faster and easier

- $\quad$ Saves time and money by bringing significant potential for cost effectively improving the time-to-market for the drug development pipeline

GLYBATOMAQ's quantum chemistry methods and software techniques

A proprietary process, using $\mathrm{AI}$ and quantum computing, generates docking studies that mathematically prove billions of binding sites to amino acids/proteins, thus carrying the capacity to both kill 
exponentially more cancer stem cells than any competitive molecule designed to date, as well as restore to full health any remaining cancer cells within one week.

This is the first software of its kind that applies AI to large-scale challenges for:

- $\quad$ Re-purposing pre-approved drugs more easily for new applications

- Empowering computational chemists to make new discoveries faster

- Comparing molecules that are much larger, allowing for more pharmaceutical advancements to cures a broader range of diseases

Benefits of A revolutionary in-silico hyper-molecule for the treatment of aggressive brain cancers

- Accelerates new target selection through the identification of "hits" to the optimization of lead compounds in the drug discovery process

- Allows researchers to simulate the behavior of a drug and support identification of the most appropriate drug dosage

- $\quad$ Predicts adverse events or possible risk of patient drop out to facilitate and optimize treatment compliance

- Enables learning from behavior in order for conversationalist bots, such as robot process assistants (RPA), to transcend reactivity by transferring and applying information to different contexts

- $\quad$ Establishes exponentially more binding sites, energy and efficacy compared to every competitive drug

- Powerfully augments efficacy of current therapies (as in the case of GLYBATOMAQ when used with the existing eleven FDA approved molecules)

\section{Competitive analysis}

Pharmaceutical companies spend hundreds of millions of dollars to design drugs and bring them to the discovery stage, often with little reassurance of viability and efficacy.

Currently, a variety of computational-intensive methods are used to review molecule matches and predict the positive effects of potential therapeutic approaches while reducing negative side effects. There is a clear opportunity for our proprietary AI quantum computing design methods to outpace current computing methods to dramatically improve patient outcomes.

Comparative studies conducted between our small molecules and other FDA approved Glioblastoma drugs have proven the supe- riority of GLYBATOMAQ. When combined with FDA approved drugs to treat the same indication, the potency of the GLYBATOMAQ molecules was amplified even further.

Figure 1 (below) displays results of the GEMDOCK study measuring fitness, energy and number of protein binding sites performed between GLYBATOMAQ and other FDA approved Glioma and Glioblastoma Cancer Drugs.

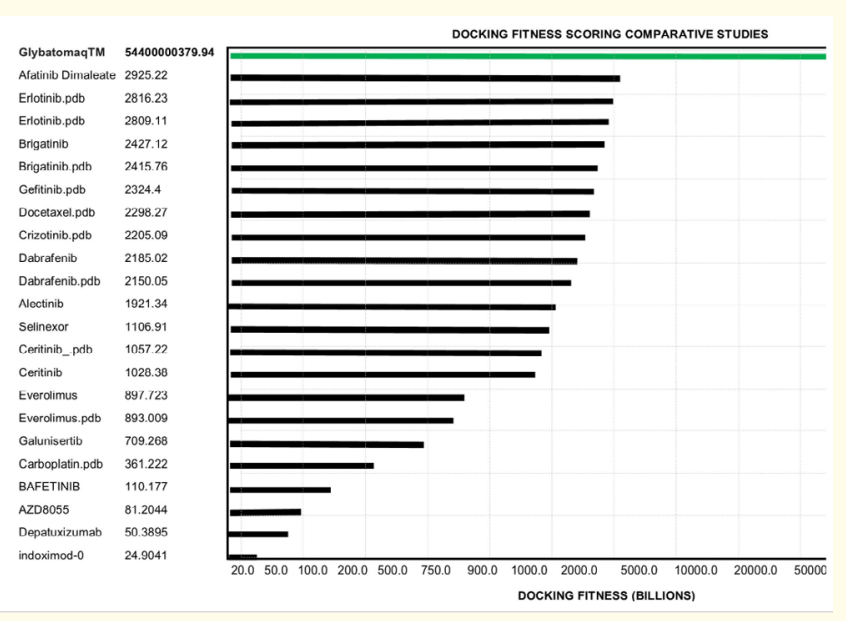

Figure 1: GLYBATOMAQ vs. Depatuxizumab - 594 million times higher binding affinities.

- $\quad$ Glybatomaq's Docking fitness $=5440000037994(5.44 \mathrm{e}+010)$

- $\quad$ Depatuxizumab Docking fitness $=50.3895$

- $\quad$ AZD8055 Docking fitness $=81.2044$

- $\quad$ BAFETINIB Docking fitness $=110.177$

- $\quad$ Galunisertib Docking fitness $=709.268$

- $\quad$ Selinexor Docking fitness $=1106.91$

- $\quad$ indoximod-0 Docking fitness $=24.9041$

- $\quad$ Alectinib.Docking fitness $=1921.34$

- $\quad$ Brigatinib Docking fitness $=2427.12$

- $\quad$ Ceritinib $=$ Docking fitness $=1028.38$

- $\quad$ Dabrafenib $=$ Docking fitness $=2185.02$

- $\quad$ Erlotinib.pdb) Docking fitness $=2809.11$

- $\quad$ Everolimus.pdb) Docking fitness $=893.009$

- $\quad$ Afatinib Dimaleate (Docking fitness $=2925.22$ )

- $\quad$ Brigatinib.pdb (Docking fitness $=2415.76$ )

- $\quad$ Carboplatin.pdb (Docking fitness = 361.222)

- $\quad$ Ceritinib_.pdb (Docking fitness $=1057.22$ )

- $\quad$ Crizotinib.pdb (Docking fitness $=2205.09$ )

- $\quad$ Dabrafenib.pdb (Docking fitness $=2150.05$ ) 
- $\quad$ Docetaxel.pdb (Docking fitness $=2298.27$ )

- $\quad$ Erlotinib.pdb (Docking fitness $=2816.23$ )

- $\quad$ Everolimus.pdb (Docking fitness $=897.723$ )

- $\quad$ Gefitinib.pdb (Docking fitness = 2324.41)

\section{Accelerating the drug development pipeline}

The second small molecule designed by the inventor is QuantumMM Microcrylaq, an in-silico hyper-molecule for the treatment of non-small cell lung cancer that possesses the same superiority profile as GLYBATOMAQ. We have the capability and proven expertise to design an almost endless supply of in-silico blockbuster molecules. Currently, there are 180 identified in the pipeline, including the recent development of small molecules to treat Alzheimer's Disease, Parkinson's Disease, for stem cell expansion, and an Opioid antagonist Molecule.

This groundbreaking technology may prove to be an invaluable strategic acquisition for any biomedical or pharmaceutical company, as it would give a critical competitive advantage of offering:

- A far more effective treatment for brain cancer and non-small cell lung tumor

- Advanced technology that repairs damaged cancer cells and dramatically improves the effectiveness of chemotherapy and radiation treatments

- The ability to produce an endless supply of Miracle Molecules

GLYBATOMAQ is clearly superior to any molecule currently treating the same indication(s), and Company A's proprietary design process can be applied successfully to hundreds of additional Miracle Molecules, generating of millions of dollars in savings in drug development for each drug that results from it. This equates to the opportunity to generate exponentially more revenue by reducing the cost of bringing to market new blockbuster drugs.

Company A has the capability to design thousands of Miracle Molecules for nearly any indication and just begun the process of seeking buyers for these in-silico molecules and proprietary drug design process.

\section{Role of galaxy AI innovations}

Galaxy AI offers GLYBATOMAQ and QuantumMM Microcrylaq as a powerful competitive edge to pharmaceutical companies that produce drugs to treat brain and lung cancer, as well as to biotech's wishing to enter this space with a proprietary blockbuster drug design method.
Its management, supported by the scientific management team of Galaxy AI's parent company, RegenConBio, LLC also have a strategic plan to bring each molecule from in-silico to the pre- clinical, pre-FDA, pre-IND (investigational new drug) development stage, adding additional value to interested buyers.

\section{Volume 3 Issue 8 August 2019}

(c) All rights are reserved by John-Ioannis Grigoriadis. 